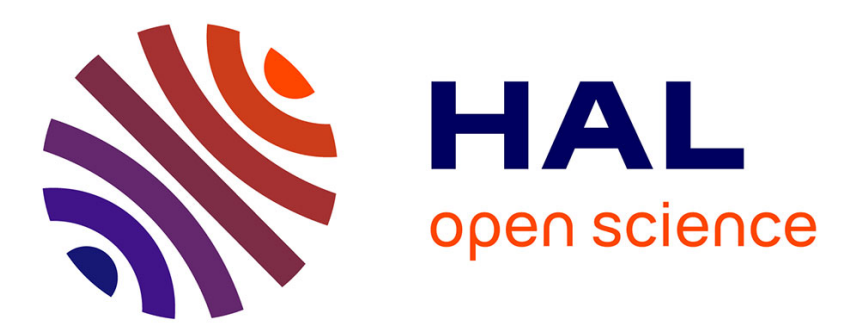

\title{
Sur l'écran et les propriétés magnétiques d'une impureté en mouvement au sein d'un métal
}

\author{
Bahram Djafari-Rouhani, P. Joyes, G. Toulouse
}

\section{To cite this version:}

Bahram Djafari-Rouhani, P. Joyes, G. Toulouse. Sur l'écran et les propriétés magnétiques d'une impureté en mouvement au sein d'un métal. Journal de Physique, 1973, 34 (8-9), pp.741-746. 10.1051/jphys:01973003408-9074100 — jpa-00207436

\section{HAL Id: jpa-00207436 https://hal.science/jpa-00207436}

Submitted on 1 Jan 1973

HAL is a multi-disciplinary open access archive for the deposit and dissemination of scientific research documents, whether they are published or not. The documents may come from teaching and research institutions in France or abroad, or from public or private research centers.
L'archive ouverte pluridisciplinaire HAL, est destinée au dépôt et à la diffusion de documents scientifiques de niveau recherche, publiés ou non, émanant des établissements d'enseignement et de recherche français ou étrangers, des laboratoires publics ou privés. 


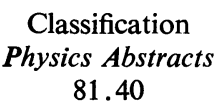

\title{
SUR L'ÉCRAN ET LES PROPRIÉTÉS MAGNÉTIQUES D'UNE IMPURETÉ EN MOUVEMENT AU SEIN D'UN MÉTAL
}

\author{
B. DJAFARI-ROUHANI, P. JOYES et G. TOULOUSE
}

\author{
Laboratoire de Physique des Solides (*), Bâtiment 510, \\ Université Paris-Sud, 91405 Orsay, France
}

(Reçu le 21 mars 1973)

\begin{abstract}
Résumé. - La charge d'écran d'une impureté chargée en mouvement dans un métal est étudiée à l'aide de la méthode des déphasages ; on suppose pour ce calcul que le potentiel self-consistent de diffusion reste inchangé par le mouvement de la particule.

On analyse ensuite, dans l'approximation Hartree-Fock, l'effet du mouvement sur la possibilité d'existence de moment magnétique localisé sur l'impureté. Le résultat de cette étude fait apparaître une tendance à la démagnétisation de l'impureté.

L'utilisation de ces résultats pour l'analyse des phénomènes d'émission ionique secondaire d'alliages de type AIM (où M est un élément de transition) est aussi évoquée.

Abstract. - The screening of a charged impurity which moves in a metal is studied by means of the phase-shift method; we suppose that the self-consistent scattering potential is not changed by the motion.

Next we analyse, in the Hartree-Fock approximation, the consequences of the motion on the possibility of existence of a magnetic moment localized on the impurity. The results show a tendency towards demagnetization of the impurity.

The implications of these results for secondary ionic emission phenomena from targets such as AlM (where $\mathrm{M}$ is a transition element) is also mentioned.
\end{abstract}

1. Introduction. - La structure électronique des alliages dilués du type $\mathrm{AlM}$, où $\mathrm{M}$ est un atome de transition, a fait l'objet de nombreux travaux [1], [2]. Chaque atome $\mathbf{M}$ possède un écran d'électrons délocalisés qui a pu être décrit à l'aide d'un hamiltonien de type Anderson [3], [4] comportant trois paramètres $E_{\mathrm{d}}, V_{\mathrm{kd}}, U$ représentant respectivement l'énergie $\mathrm{du}$ niveau atomique $\mathrm{d}$, son couplage au continuum et l'énergie d'interaction coulombienne intra-atomique.

Nous nous intéresserons ici à la manière dont évolue cet écran lorsque l'atome $M$ se meut dans l'alliage. Ce problème est intéressant à divers titres ; il est important en particulier pour l'étude de l'émission secondaire de cibles du type AlM soumises à un bombardement d'ions primaires [5] où l'on a besoin de connaître l'état électronique de la particule $\mathbf{M}$ lorsqu'elle émerge à la surface de la cible.

L'application que nous développerons dans cet article porte sur les propriétés magnétiques de l'im-

(*) Laboratoire associé au Centre National de la Recherche Scientifique. pureté. Ces propriétés sont régies essentiellement par le terme coulombien; ainsi, dans une théorie de champ moyen, il existera un moment magnétique localisé sur $\mathrm{M}$ si :

$$
n\left(E_{\mathrm{F}}\right) U>1
$$

où $n\left(E_{\mathrm{F}}\right)$ est la densité d'état du niveau lié virtuel au niveau de Fermi.

Restant constamment dans le cadre d'une approximation de vitesses faibles, on étudiera, dans la première partie, l'écran d'une particule chargée en mouvement dans un métal puis, dans une deuxième partie, les conséquences de ce mouvement sur le magnétisme d'impureté (approximation de champ moyen).

2. Ecran d'une particule chargée en mouvement. 2.1 CalCUl GÉNÉRAL. - On va étendre au cas d'une particule chargée en mouvement (de vitesse v) la méthode des déphasages [1], [7] appliquée jusqu'alors à l'étude de l'écran d'une impureté statique dans un métal (considéré comme un gaz d'électrons libres). On se limitera aux vitesses $\mathbf{v}$ inférieures à la vitesse 
de Fermi ce qui correspond, pour un atome de fer dans l'aluminium, par exemple, à une énergie cinétique $\lesssim 1 \mathrm{keV}$.

Le nombre d'états déplacés par l'introduction de la perturbation à travers une surface quelconque $S$ de l'espace réciproque, c'est-à-dire le flux du courant de la densité d'états à travers une telle surface s'écrit :

$$
N=-2 \Sigma_{l}(2 l+1) \int_{S} \mathbf{J}_{l} \cdot \mathrm{d} \mathbf{S}
$$

Dans cette expression $\mathbf{J}_{l}$ est le courant de la densité d'état dans l'espace réciproque correspondant à la valeur $l \mathrm{du}$ moment angulaire, le signe négatif est dû à la convention de normale extérieure.

Considérons d'abord le cas de l'impureté statique. La densité d'état tridimensionnelle dans l'espace réciproque (Fig. 1) s'écrit :

$$
\rho(\mathbf{q})=\frac{R}{4 \pi^{2} q^{2}}
$$

(où $R$ est le rayon du gaz d'électrons) par ailleurs, pour une valeur $l$ du moment angulaire, l'effet de la perturbation [7] est de déplacer un point de vecteur d'onde $\mathbf{q}$ en $\mathbf{q}-\delta_{l}(q) \mathbf{q} / R q$, on en déduit :

$$
J_{l}=-\frac{\mathbf{q} \delta_{l}(q) R}{q R 4 \pi^{2} q^{2}}
$$

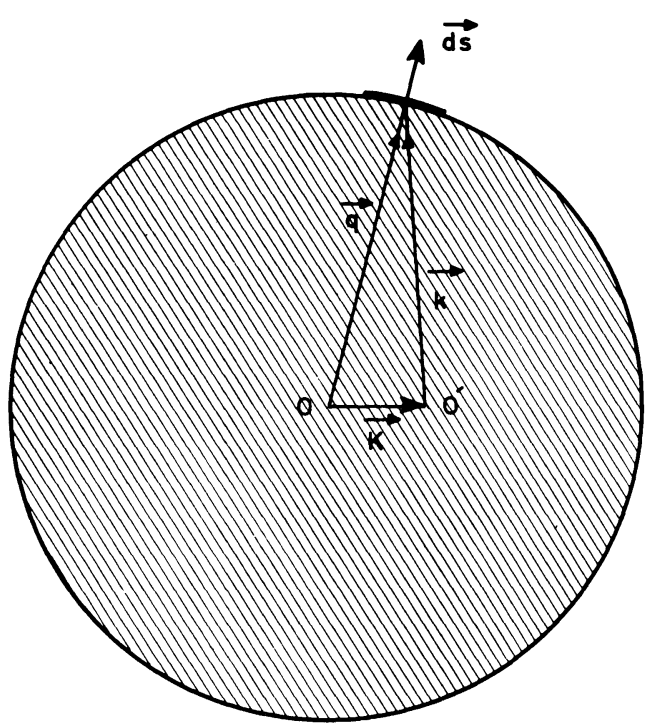

FIg. 1. - Référentiel fixe (origine en $O$ ) et référentiel lié à la particule (origine en $O^{\prime}$ ) dans l'espace réciproque.

L'éq. (2) permet de calculer aisément le nombre d'états déplacés à travers une sphère de centre $O$ et de rayon $q=q_{0}$ (en particulier, pour la sphère de Fermi, $q_{0}=k_{\mathrm{F}}$ ), on retrouve, comme il se doit, la règle de somme de Friedel :

$$
N=\frac{2}{\pi} \Sigma_{l}(2 l+1) \delta_{l}\left(q_{0}\right)
$$

Considérons maintenant l'impureté munie d'une vitesse uniforme $\mathbf{v}=\hbar \mathbf{K} / m$ ( $m=$ masse de l'électron), à cause de la symétrie sphérique de la distribution de vitesses des électrons de conduction, seul le module de $\mathbf{v}$ va intervenir dans les calculs. Au lieu de se placer dans un référentiel fixe où la particule a la vitesse $\mathbf{v}$, il est plus commode de se placer dans le référentiel de la particule où les électrons sont munis dans leur ensemble d'une vitesse moyenne $-\mathbf{v}$; dans ce cas, la surface de Fermi reste une sphère mais déplacée de $-\mathbf{K}$ dans l'espace réciproque (Fig. 1).

Alors, de façon analogue au cas statique, on peut écrire :

$$
\mathbf{J}_{l}=-\frac{\mathbf{k} \delta_{l}(k) R}{k R 4 \pi^{2} k^{2}}
$$

où $\mathbf{k}=\mathbf{q}-\mathbf{K}$.

Le nombre d'états déplacés à travers une sphère $q=q_{0}$, centrée sur $O$, correspondant à l'énergie $E=\hbar^{2} q_{0}^{2} / 2 m$ (comptée à partir du bas de la bande de conduction se déduit de (2) en utilisant (3). Se limitant à un développement limité à l'ordre 2 en $K / q_{0}\left(K \ll q_{0}\right)$, on aboutit à (voir appendice) :

$$
\begin{aligned}
N(E)=\frac{2}{\pi} \Sigma_{l}(2 l+1) \times & \\
& \times\left\{\delta_{l}(E)-\frac{E_{\mathrm{c}}}{3}\left(\dot{\delta}_{l}(E)-2 E \ddot{\delta}_{l}(E)\right)\right\}
\end{aligned}
$$

avec

$$
E_{\mathrm{c}}=\frac{1}{2} m v^{2}=\frac{1}{2} M v^{2} \frac{m}{M}=\frac{\hbar^{2} K^{2}}{2 m}
$$

( $M$ : masse de la particule), $\dot{\delta}_{i}, \ddot{\delta}_{\imath}$ sont les dérivées successives de $\delta_{i}$ par rapport à l'énergie.

La dérivation de (4) donne la variation de la densité d'état due à l'introduction de l'impureté

$$
\begin{aligned}
n(E)=\frac{\partial N(E)}{\partial E}=\frac{2}{\pi} \Sigma_{l}(2 l+1) \times & \\
& \times\left\{\dot{\delta}_{l}+\frac{E_{\mathrm{c}}}{3}\left(\ddot{\delta}_{l}+2 E \ddot{\delta}_{l}\right)\right\} .
\end{aligned}
$$

2.2 RÉSUlTATS ET DISCUSSION DANS LE CAS D'UNE RÉSONANCE LORENTZIENNE ET DANS LE CAS U $=0$. Pour simplifier on se limitera ici à un seul niveau de moment angulaire $l$ (le facteur de dégénérescence $2(2 l+1)$ est supprimé $)$ et on considérera le cas d'une résonance lorentzienne

$$
\delta=\frac{\Delta}{\left(E-E_{0}\right)^{2}+\Delta^{2}}
$$

où $E_{0}=E_{\mathrm{d}}$ donne la position du niveau lié virtuel et $\triangle$ sa largeur.

On se propose d'étudier l'effet du mouvement de la particule sur la densité d'états $n(E) ; z=E-E_{0}$ sera pris comme variable, $\Delta$ et $E_{0}$ comme paramètres. 
L'expression (5) de $n(E)$ se met sous la forme :

$$
\begin{aligned}
n(E)= & \frac{\Delta}{\pi\left(z^{2}+\Delta^{2}\right)}\left[1+\frac{2 E_{c}}{3\left(z^{2}+\Delta^{2}\right)^{2}} \times\right. \\
& \left.\left(5 z^{3}+6 E_{0} z^{2}-3 \Delta^{2} z-2 \Delta^{2} E_{0}\right)\right] .
\end{aligned}
$$

Le premier terme du crochet est le terme statique, l'effet de la vitesse est contenu dans le second terme.

La figure 2 donne une allure schématique de $n(E)$; on note que le maximum de $n(E)$ se produit, au premier ordre en $E_{\mathrm{c}}$, pour $z=-E_{\mathrm{c}}$ et que l'effet du mouvement est, pour $|z|<\Delta \sqrt{3} / 3$ (de l'ordre de la largeur du niveau lié virtuel), de diminuer la densité d'état et, pour $|z|>\Delta \sqrt{3} / 3$ de l'augmenter.

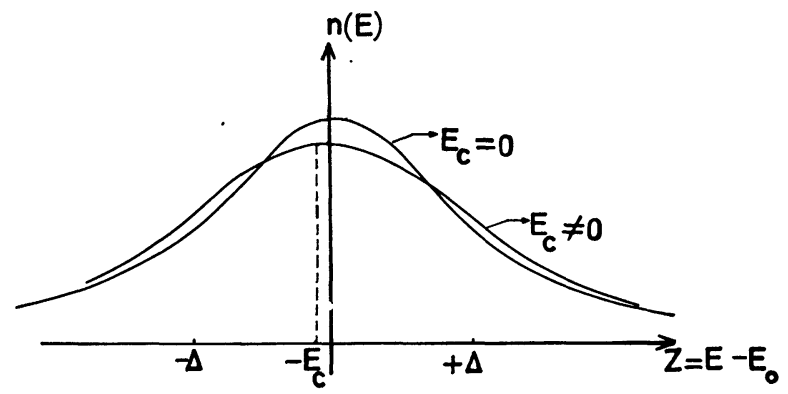

Fig. 2. - Allure schématique de la densité d'états pour $E_{\mathrm{c}}=0$ (lorentzienne) et pour $E_{\mathrm{c}} \neq 0$.

Signalons enfin que le choix d'une densité d'état gaussienne pour l'état lié virtuel conduit à des conclusions analogues aux précédentes.

Une explication qualitative de nos résultats apparaît figure 3. Dans l'espace réciproque, la zone comprise entre les sphères $E_{0}-\Delta$ et $E_{0}+\Delta$ représente la région où des états supplémentaires sont apparus du fait de l'état lié virtuel. Les sphères $E_{\mathrm{S}}$ et $E_{\mathrm{D}}$ représentent le lieu des points d'énergies $E$ où l'on veut mesurer la densité d'état en régime statique $E_{\mathrm{S}}$ et en régime dynamique $E_{\mathrm{D}}$. Dans la figure $3 a$, correspondant à $E_{0}-\Delta \lesssim E_{\mathrm{S}} \lesssim E_{0}+\Delta$ on voit que la sphère $E_{\mathrm{S}}$ est, au total, plongée dans une région plus dense que $E_{\mathrm{D}}$, d'où diminution de densité d'état due au mouvement. Dans $3 b\left(E_{\mathrm{S}} \lesssim E_{0}-\Delta\right)$ et $3 c\left(E_{\mathrm{S}} \gtrsim E_{0}+\Delta\right)$ apparaît l'effet inverse.

$\mathrm{La}$ discussion de la variation du nombre total d'états

$$
\begin{aligned}
& N_{E_{\mathrm{c}}}- N_{E_{\mathrm{c}}=0}=-\frac{E_{\mathrm{c}}}{3 \pi}\left[\dot{\delta}\left(E_{\mathrm{F}}\right)-2 E_{\mathrm{F}} \ddot{\delta}\left(E_{\mathrm{F}}\right)\right]= \\
&=-\frac{E_{\mathrm{c}}}{3 \pi} \frac{\Delta}{\left(Z_{\mathrm{F}}^{2}+\Delta^{2}\right)^{2}}\left(Z_{\mathrm{F}}^{2}+4 E_{\mathrm{F}} Z_{\mathrm{F}}-\Delta^{2}\right)
\end{aligned}
$$

peut aussi s'avérer intéressante dans certains problèmes. Pour toutes les valeurs de $Z_{\mathrm{F}}=E_{\mathrm{F}}-E_{0}$ où l'on peut dire que le niveau de Fermi tombe dans la zone d'énergies du niveau lié virtuel $\left(\left|Z_{\mathrm{F}}\right|\right.$ de l'ordre de quelques $\Delta$ ), l'expression 7 considérée

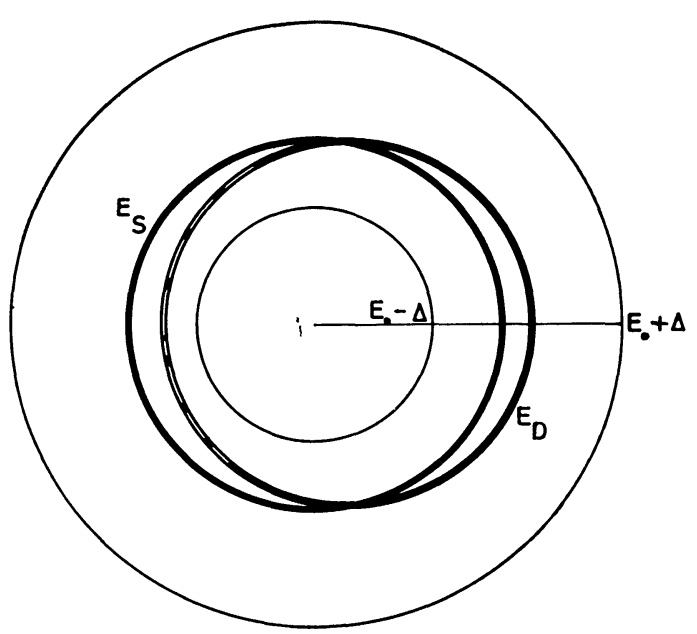

a

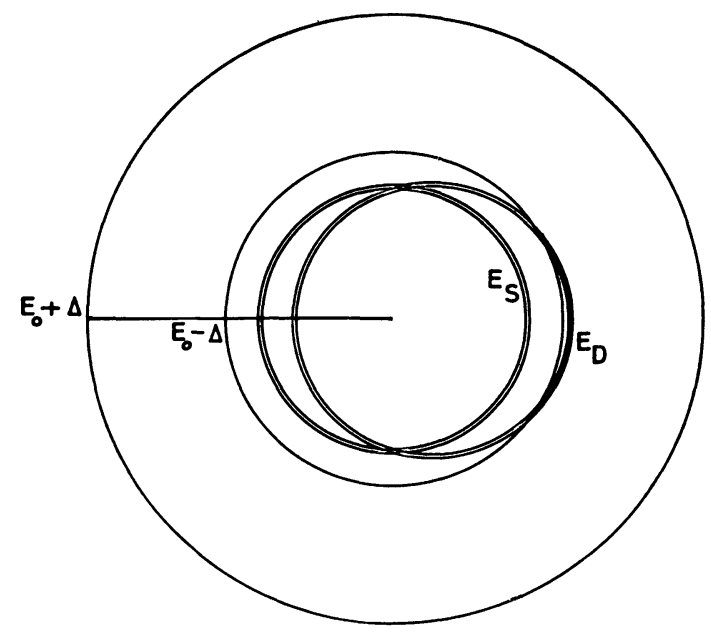

b

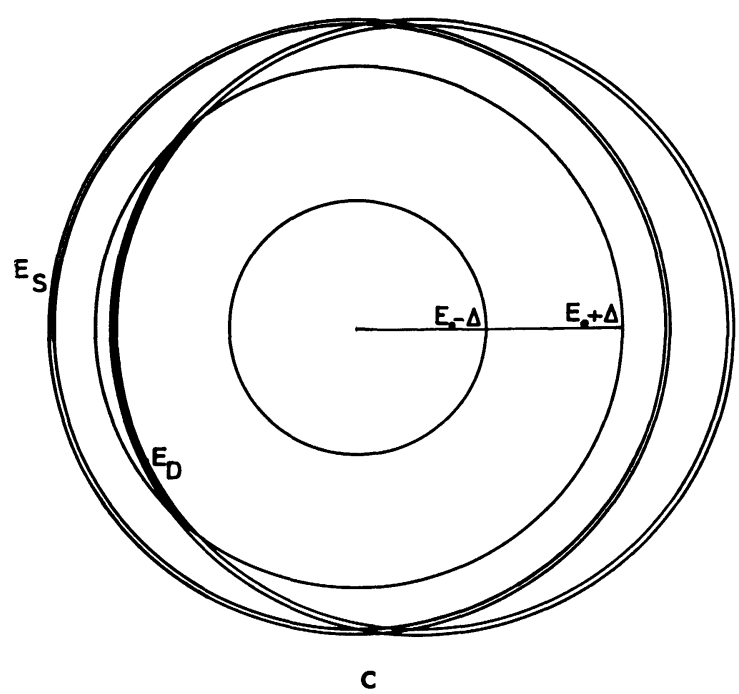

Fig. 3. - La zone comprise entre les sphères $E_{0}-\Delta$ et $E_{0}+\Delta$ est la région où des états supplémentaires sont apparus du fait de l'état lié virtuel. Les sphères $E_{\mathrm{S}}$ et $E_{\mathrm{D}}$ représentent les lieux des points d'énergie $E$ où l'on veut mesurer la densité d'états en régime statique $\left(E_{\mathrm{S}}\right)$ et en régime dynamique $\left(E_{\mathrm{D}}\right)$ : a) cas $\left.E_{0}-\Delta \lesssim E_{\mathrm{S}} \lesssim E_{0}+\Delta ; b\right)$ cas $E_{\mathrm{S}} \lesssim E_{0}-\Delta$; c) cas $E_{\mathrm{S}} \gtrsim E_{0}+\Delta$. 
comme fonction de $Z_{\mathbf{F}}$ est (au premier ordre en $\left.\Delta / E_{\mathrm{F}}\right)$

- positive si $Z_{\mathrm{F}}<0$ c'est-à-dire $E_{0}>E_{\mathrm{F}}$;

- négative si $Z_{\mathrm{F}}>0$ c'est-à-dire $E_{0}<E_{\mathrm{F}}$.

L'effet de la vitesse est donc d'augmenter la charge d'écran si $E_{0}>E_{\mathrm{F}}$, de la diminuer dans le cas inverse.

Le défaut ou l'excès d'écran que donne le résultat précédent est obtenu moyennant l'hypothèse que le potentiel self-consistent de diffusion est inchangé par le mouvement de la particule : les paramètres $E_{0}$ et $\Delta$ sont supposés indépendants de $E_{\mathrm{c}}$.

Il pourrait sembler plus raisonnable de supposer que l'écran reste parfait tandis que le potentiel self- consistent se réajuste. En théorie de la réponse linéaire [8] et pour les faibles vitesses, l'impureté induit dans le gaz d'électrons un courant dipolaire (backflow) de sorte que le flux total de la charge à travers un plan perpendiculaire à la vitesse reste nul. La distribution de ce courant a une symétrie correspondant à un moment angulaire $l=1$. On peut alors penser que le réajustement éventuel du potentiel selfconsistent porte essentiellement sur le déphasage $l=1$. On envisage ici le cas d'atomes de transition et on supposera donc que les paramètres $E_{0}$ et $\Delta$ correspondant à la résonance $d(l=2)$ sont indépendants de $E_{\mathrm{c}}$.

3. Evolution des propriétés magnétiques avec la vitesse v. Transition magnétique-non magnétique. Nous considérons maintenant le problème des conditions d'existence d'un moment magnétique localisé sur une impureté en mouvement dans un alliage et plus particulièrement l'effet de la vitesse sur la courbe de transition magnétique-non magnétique [6].

Avec les notations

$x=\frac{E_{\mathrm{F}}-E_{\mathrm{d}}}{U}=\frac{E_{\mathrm{F}}-E_{0}}{U}+N=\frac{Z_{\mathrm{F}}}{U}+N \quad y=\frac{\pi \Delta}{U}$,

où $E_{0}=E_{\mathrm{d}}+U N$ et $N$ est le nombre d'électrons du niveau lié pour une direction de spin, la courbe de transition magnétique-non magnétique est définie, pour $E_{\mathrm{c}}=0$, par le système d'éq. (6) :

$$
\left.\begin{array}{l}
\frac{y}{\pi} \operatorname{cotg} \pi N=N-x \\
\frac{y}{\pi^{2}(x-N)^{2}+y^{2}}=1
\end{array}\right\} .
$$

La première équation fournit $N$ pour $x$ et $y$ (c'est-à-dire $\left.E_{\mathrm{F}}, E_{\mathrm{d}}, U\right)$ donnés et la deuxième exprime l'égalité :

$$
n\left(E_{\mathrm{F}}\right) U=1 \text {. }
$$

Ce système est équivalent à un autre, plus simple, où $x$ et $y$ sont définis comme fonctions de $N$

$$
\left.\begin{array}{c}
N-x=\frac{\sin (\pi N) \cos (\pi N)}{\pi} \\
y=\sin ^{2}(\pi N) .
\end{array}\right\}
$$

Lorsque $E_{\mathrm{c}}$ est différent de zéro, on aboutit à un système du même type :

$$
\begin{array}{r}
N-x=\frac{y}{\pi} \operatorname{cotg}\left\{\pi N+\frac{\pi E_{\mathrm{c}}}{3 U} \frac{y}{\left[\pi^{2}(x-N)^{2}+y^{2}\right]^{2}} \times\right. \\
\left.\times\left[\pi^{2}(x-N)^{2}+\frac{4 \pi^{2} E_{\mathrm{F}}}{U}(x-N)+y^{2}\right]\right\} \\
\frac{y}{\pi^{2}(x-N)^{2}+y^{2}}\left\{1-\frac{2 \pi E_{\mathrm{c}}}{3 U} \frac{1}{\left(\pi^{2}(x-N)^{2}+y^{2}\right)^{2}} \times\right. \\
\times\left[\pi^{3}(x-N)^{3}-\frac{6 \pi^{3} E_{\mathrm{F}}}{U}(x-N)^{2}+\right. \\
\left.\left.\quad+\pi y^{2}(x-N)+\frac{2 \pi E_{\mathrm{F}}}{U} y^{2}\right]\right\}=1
\end{array}
$$

La première éq. de (9) se déduit de (7) et de la première éq. de $(8)$; la deuxième exprime $n\left(E_{\mathrm{F}}\right) U=1$.

Au premier ordre en $E_{\mathrm{c}}$ et si l'on se place dans une région où $N$ est différent de 0 ou 1 (auquel cas $\sin ^{2} \pi N \neq 0$ ) on peut développer la première éq. de (9) :

$$
\begin{aligned}
N-x= & \frac{y}{\pi}\left\{\operatorname{cotg}(\pi N)-\frac{1}{\sin ^{2} \pi N} \times\right. \\
& \times \frac{\pi E_{\mathrm{c}} y}{3 U\left[\pi^{2}(x-N)^{2}+y^{2}\right]^{2}} \\
& \left.\times\left[\pi^{2}(x-N)^{2}+\frac{4 \pi^{2} E_{\mathrm{F}}}{U}(x-N)+y^{2}\right]\right\} .
\end{aligned}
$$

On ne cherchera pas à tracer exactement la courbe 9 , seule son allure sera donnée par la recherche de points caractéristiques.

Aux points d'intersection 8 et 9 on a évidemment les mêmes valeurs de $x$ et $y$ mais des valeurs différentes $N_{\mathrm{S}}$ et $N_{\mathrm{D}}\left(N_{\mathrm{S}}\right.$ correspond à $8:$ régime statique, $N_{\mathrm{D}}$ à 9 : régime dynamique) pour $N$. On écrira :

$$
N_{\mathrm{D}}=N_{\mathrm{s}}+\delta N
$$

où $\delta N$ sera le terme du premier ordre du développement en $E_{\mathrm{c}}$ de $N_{\mathrm{D}}$. En tenant compte de 8, et après simplifications on obtient :

$$
\begin{aligned}
& 2 \delta N=-\frac{E_{\mathrm{c}}}{3 U} \frac{1}{\sin \left(\pi N_{\mathrm{S}}\right)} \times \\
& \times\left(\sin \left(\pi N_{\mathrm{S}}\right)-\frac{4 \pi E_{\mathrm{F}}}{U} \cos \left(\pi N_{\mathrm{S}}\right)\right) \\
& 2 \delta N=\frac{-2 E_{\mathrm{c}}}{3 U \sin \left(\pi N_{\mathrm{S}}\right) \cos \left(\pi N_{\mathrm{s}}\right)} \times \\
& \times\left(-\sin \left(\pi N_{\mathrm{S}}\right) \cos \left(\pi N_{\mathrm{S}}\right)-\frac{6 \pi E_{\mathrm{F}}}{U} \cos ^{2}\left(\pi N_{\mathrm{S}}\right)+\right. \\
& \left.+\frac{2 \pi E_{\mathrm{F}}}{U} \sin ^{2}\left(\pi N_{\mathrm{S}}\right)\right) \\
& y=\sin ^{2} \pi N_{\mathrm{S}} \\
& N_{\mathrm{S}}-x=\frac{\sin \left(\pi N_{\mathrm{S}}\right) \cos \left(\pi N_{\mathrm{S}}^{7}\right)}{\pi}
\end{aligned}
$$


Les deux premières équations donnent :

$$
\frac{4 \pi E_{\mathrm{F}}}{U} \operatorname{tg}^{2}\left(\pi N_{\mathrm{S}}\right)-3 \operatorname{tg}\left(\pi N_{\mathrm{S}}\right)+\frac{8 \pi E_{\mathrm{F}}}{U}=0
$$

ou encore

$$
\operatorname{tg} \pi N_{\mathrm{S}}=\frac{3 \pm \sqrt{9+8\left(\frac{4 \pi E_{\mathrm{F}}}{U}\right)^{2}}}{2 \frac{4 \pi E_{\mathrm{F}}}{U}}
$$

En reportant ces deux valeurs de $N_{\mathrm{S}}$ dans les deux dernières équations de 10 on obtient les coordonnées des deux points d'intersection de 8 et 9 . A titre indicatif, pour $4 \pi E_{\mathbf{F}} / U \gg 1$

$$
\begin{aligned}
\operatorname{tg} \pi N_{\mathrm{S}} & \sim \pm \sqrt{2} \\
y & \sim \frac{2}{3} \\
x & \sim 0,15 \text { et } 0,85 .
\end{aligned}
$$

Un autre point caractéristique de la courbe 9 est celui correspondant à $x=\frac{1}{2}$. Comme plus haut, on pose en ce point

$$
\begin{aligned}
& y_{\mathrm{D}}=1+\delta y \\
& N_{\mathrm{D}}=\frac{1}{2}+\delta N
\end{aligned}
$$

et la deuxième éq. (9) donne au premier ordre en $E_{\mathrm{c}}$

$$
(1-\delta y)\left(1-\frac{2 \pi E_{\mathrm{c}}}{3 U} \frac{2 \pi E_{\mathrm{F}}}{U}\right)=1
$$

d'où

$$
\delta y=-\frac{4 \pi^{2}}{3} \frac{E_{\mathrm{c}} E_{\mathrm{F}}}{U^{2}} .
$$

On remarque que cette correction (en $E_{\mathrm{c}} E_{\mathrm{F}} / U^{2}$ ) est négative ce qui permet de donner figure 4 l'allure de

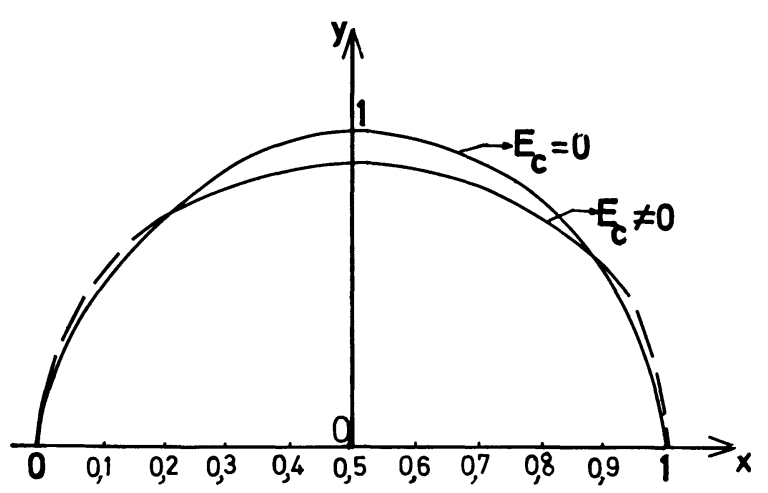

Fig. 4. - Allure schématique de la courbe de transition de phase magnétique-non magnétique pour $E_{\mathrm{c}}=0$ (Fig. 4 de la réf. [6]) et pour $E_{\mathrm{c}} \neq 0$. la courbe 9 de transition magnétique-non magnétique en régime dynamique. D'une façon générale, la conclusion est que le mouvement de $\mathrm{M}$ a pour effet de produire un rétrécissement de la zone magnétique dans la partie centrale de la courbe et un élargissement sur les côtés.

4. Conclusion. - Nos calculs ont été faits pour un domaine de vitesses faibles $\left(E_{\mathrm{c}} / E_{\mathrm{F}} \ll 1\right)$. Cette approximation est justifiée par exemple pour l'étude de l'émission ionique secondaire (approche de la surface). L'extension au domaine de vitesses fortes soulèverait sans doute des problèmes délicats, en particulier concernant l'hypothèse de constance des paramètres $E_{\mathrm{d}}, \Delta$ et $U$.

Notre résultat essentiel est que, dans la mesure où l'approximation Hartree-Fock peut rendre compte qualitativement des tendances, l'effet de la vitesse est de rendre en général plus difficile l'apparition du magnétisme. Ceci se traduit par le fait que dans un diagramme $x, y, x=\left(E_{\mathrm{F}}-E_{\mathrm{d}}\right) / U$ et $y=\pi \Delta / U$, la zone limitée par la courbe de transition magnétiquenon magnétique est rétrécie. Ainsi une impureté statique magnétique peut se démagnétiser lorsqu'on lui imprime un certain mouvement.

Cette conclusion qui contribue à simplifier la description d'une impureté magnétique lorsqu'elle est en mouvement sera exploitée ultérieurement, en particulier dans une étude théorique de l'émission ionique secondaire.

\section{Appendice}

L'intégrale (2) à calculer peut s'écrire avec les notations de la figure 1 :

$$
\begin{aligned}
N(E)=\frac{2}{\pi} \Sigma_{l}(2 l & +1) \int_{\text {Surface }|\mathbf{q}|=q_{0}} \\
& \times \frac{\mathbf{k} \delta_{l}(k) R}{k R 4 \pi^{2} k^{2}} q_{0}^{2} \sin \theta \mathrm{d} \theta \mathrm{d} \varphi \frac{\mathbf{q}}{q_{0}}
\end{aligned}
$$

où

et

$$
\mathbf{k}=\mathbf{q}-\mathbf{K}
$$

$$
E=\frac{\hbar^{2} q_{0}^{2}}{2 m}
$$

Ayant

$$
\mathbf{k} \cdot \mathbf{q}=q_{0}^{2}-\mathbf{q} \cdot \mathbf{K}=q_{0}^{2}-K q_{0} \cos \theta
$$

et

$$
k=|\mathbf{k}|=\sqrt{q_{0}^{2}}-2 K q_{0} \cos \theta+K^{2}
$$

on peut développer $\mathbf{k . q} / k^{3}$ au second ordre en $K / q_{0}$.

Par ailleurs on effectue le développement limité suivant pour $\delta_{i}(k)$ 


$$
\begin{aligned}
\delta_{l}(k)=\delta_{l}\left(q_{0}\right)+\delta_{l}^{\prime}\left(q_{0}\right)(k- & \left.q_{0}\right)+ \\
& +\delta_{l}^{\prime \prime}\left(q_{0}\right)\left(\frac{k-q_{0}}{2}\right)^{2}+\cdots \\
=\delta_{l}\left(q_{0}\right)+\delta_{l}^{\prime}\left(q_{0}\right)[- & \left.K \cos \theta+\frac{K^{2}}{2 q_{0}} \sin \theta\right] \\
& +\delta_{l}^{\prime \prime}\left(q_{0}\right) \frac{K^{2} \cos ^{2} \theta}{2}+\theta\left(k^{2}\right)
\end{aligned}
$$

où $\delta_{l}^{\prime}, \delta_{l}^{\prime \prime}, \ldots$ sont les dérivées successives de $\delta_{l}$ par rapport au module du vecteur d'onde.
On peut alors facilement calculer (A.1) au second ordre en $K / q_{0}$ sous forme :

$$
\begin{aligned}
N(E) & =\frac{2}{\pi} \Sigma_{l}(2 l+1) \times \\
& \times\left\{\delta_{l}\left(q_{0}\right)-\frac{K^{2}}{3 q_{0}}\left[\delta_{l}^{\prime}\left(q_{0}\right)-\frac{q_{0}}{2} \delta_{l}^{\prime \prime}\left(q_{0}\right)\right]\right\} .
\end{aligned}
$$

Il est préférable d'utiliser les dérivées $\dot{\delta}_{l}, \ddot{\delta}_{l}, \ldots$ de $\delta_{l}$ par rapport à l'énergie $E$.

Utilisant l'expression de $E$ en fonction de $q_{0}$ on peut établir les nouvelles dérivées et déduire de (A.2) l'expression (4) de $N(E)$.

\section{Bibliographie}

[1] Friedel, J., Nuovo Cimento, Supplemento VII (1958) 287.

[2] Friedel, J., J. Physique 23 (1962) 692.

[3] Joyes, P. et Toulouse, G., Phys. Lett. 39A (1972) 267.

[4] JoYes, P., J. Physique 30 (1969) 365.

[5] Blaise, G. et Slodzian, G., J. Physique 31 (1970) 93.
[6] Anderson, P. W., Phys. Rev. 124 (1961) 41.

[7] Friedel, J., Phil. Mag. 43 (1952) 153.

[8] PINES, D. et NozIÈres, P., The theory of quantum liquids (W. A. Benjamin, INC, New York, Amsterdam), 1966, p. 172. 